Szabo, Fabrice. " "The Bishop's Candlesticks" : une certaine tradition de la réception hollywoodienne des Misérables. » Nouvelle Revue Synergies Canada, N¹3 (2020)

\title{
"The Bishop's Candlesticks » : une certaine tradition de la réception hollywoodienne des Misérables
}

\author{
Fabrice Szabo \\ Université de Windsor \\ Canada
}

II est difficile de saisir l'essence des Misérables, de vouloir la résumer en quelques mots, en quelques idées fortes, en quelques personnages, en quelques objets, et c'est bien sûr cette dernière dimension qui sera au centre de notre article. Le roman est à la fois policier et utopique, c'est une épopée et une idylle, l'histoire d'un homme et le destin de quelques idées. Comme la barricade des insurgés, le roman est le fruit d'une économie circulaire, dirait-on aujourd'hui. II a été parfois (trop souvent ?) reproché à ce roman cette facilité : il est bancal, fait de bric et de broc, un «fourre-tout » excessif. Est-il nécessaire pour le lecteur de connaître les méandres de la vie de couvent ou de la construction des égouts de Paris ? On aurait tort d'y voir que des débordements verbeux et encyclopédisme bigarré. Hugo étreint large mais il étreint bien.

C'est parce que Victor Hugo comprend qu'un roman-portrait de son siècle ne peut avoir qu'une seule facette, le texte ne peut se limiter à être une surface plane et réfléchissante. Le roman doit être fait de gouffres et d'aspérités. L'œil ne peut en saisir immédiatement toute la portée. Au contraire le roman ouvre de multiples possibilités de réceptions et d'adaptations, il se fait kaléidoscope ; l'éclatement du regard élargit plus qu'il ne fractionne comme le remarque Guy Rosa : "Cette amplitude exceptionnelle importe. Elle situe les conditions, les langages, les modes de vie et les préoccupations les uns par rapport aux autres [...] ; surtout, elle reproduit ce caractère essentiel d'une société qui est son extrême variété et sa vastitude : le fait qu'elle offre au regard un horizon illimité » (2).

Et pourtant dans cet infiniment grand, il faut de l'infiniment petit. II faut qu'aux personnages s'accrochent et s'attachent des objets : Thénardier ne semble jamais vouloir se séparer de la peinture du "Sergent de Waterloo »; Cosette s'accroche à sa poupée ; Marius règle sa vie sur le souvenir de son père incarné dans une lettre-relique ; Valjean a pour talisman les chandeliers de l'évêque Myriel. Ce sont des objets de transaction tout autant que de transition : l'acquisition de la poupée est le prélude au rachat de la fillette par Valjean, la lettre paternelle conservée par Marius va l'obliger à un difficile partage entre ce qu'il doit donner par amour filial et ce qu'il doit donner par amour passionnel, le don des chandeliers est la première étape de la rédemption du bagnard. Dans cette civilisation matérielle naissante, il est même une entité faite d'objets, un amas fait conscience : la barricade des insurgés. Cette barricade prendra à l'occasion une dimension proprement monstrueuse comme c'est le cas dans la dernière adaptation cinématographique américaine des Misérables, ${ }^{1}$ où elle boucle le récit en reprenant la figure du monstre marin que Valjean et ses compagnons de bagne extraient de l'océan dans l'ouverture du film.

Notre projet pour cet article est de nous intéresser en particulier à un «objet-clé » du roman : les fameux " chandeliers de l'évêque ». En effet, dans plusieurs adaptations cinématographiques du roman, ces chandeliers objets de transition/transaction- que Myriel a confiés à Jean Valjean apparaissent à chaque fois que le héros est confronté à un dilemme, comme s'ils étaient la manifestation concrète de sa conscience. Ces "Chandeliers de l'évêque » ont été parfois des substituts si puissants au personnage du bagnard qu'ils deviennent le titre alternatif du roman. Dans cet article, nous verrons comment « The Bishop's Candelsticks » ont désigné l'œuvre hugolienne dans la tradition " originale » de la réception hollywoodienne des Misérables. Prenant appui sur la redécouverte récente d'un film de court-métrage d'Albert Capellani, Le Chemineau, nous allons chercher à montrer comment les chandeliers de Monseigneur Myriel ont été les « objets phares » de certaines adaptations tout autant que la barricade des révolutionnaires l'a été dans certaines autres. Le choix fait est évidemment significatif d'une lecture, d'un contexte culturel et politique de production : une adaptation c'est ce que l'on retient et ce que l'on retranche. A ce titre, nous nous intéresserons particulièrement à l'adaptation méconnue réalisée par Lewis Milestone en 1953. Nous verrons que, dans cette œuvre, l'omniprésence des chandeliers fait que le roman devient avant tout le récit d'une rédemption individuelle dans laquelle la mystique chrétienne est fortement associée à l'image du self-made man.

\section{Du Chemineau d'Albert Capellani aux Misérables de Lewis Milestone}

Afin de mener à bien cette étude, il nous faut procéder à un retour sur la mémoire des adaptations des Misérables. En effet, ces œuvres cinématographiques se répondent les unes aux autres, nul - qu'il soit scénariste, réalisateur ou producteur - ne peut ignorer ses prédécesseurs. 
La tradition des adaptations des Misérables remonte à cet événement fondateur - et oublié - que fut le premier film d'Albert Capellani pour Pathé et intitulé Le Chemineau en 1905. Ce court-métrage fut aussi exploité aux États-Unis à partir de 1906 sous deux titres: The Tramp et The Strong Arm of the Law. Avant que ce film ne soit retrouvé et restauré, il était souvent considéré que c'était là une adaptation du drame de Jean Richepin. ${ }^{2}$ En effet, en 1913 Capellani offrit une adaptation d'un autre drame de Jean Richepin, La Glu. Tout le monde avait aussi à l'esprit sa fameuse adaptation des Misérables de 1913, ${ }^{3}$ et en l'absence de copies visibles, le lien avec ce Chemineau de 1905 n'avait pas été fait. Et pourtant, dans cette courte bande, le cinéaste présente en quelques séquences le célèbre épisode de l'arrivée d'un Jean Valjean hirsute dans une petite ville hostile, puis sa rencontre avec Myriel, un évêque qu'il va nuitamment dépouiller de quelques pièces de son argenterie avant de prendre la fuite. Ce petit film de 5 minutes se conclut par l'arrestation de Jean Valjean. C'est notre premier regard sur un Jean Valjean de cinéma et c'est aussi son premier regard vers la caméra et les spectateurs.

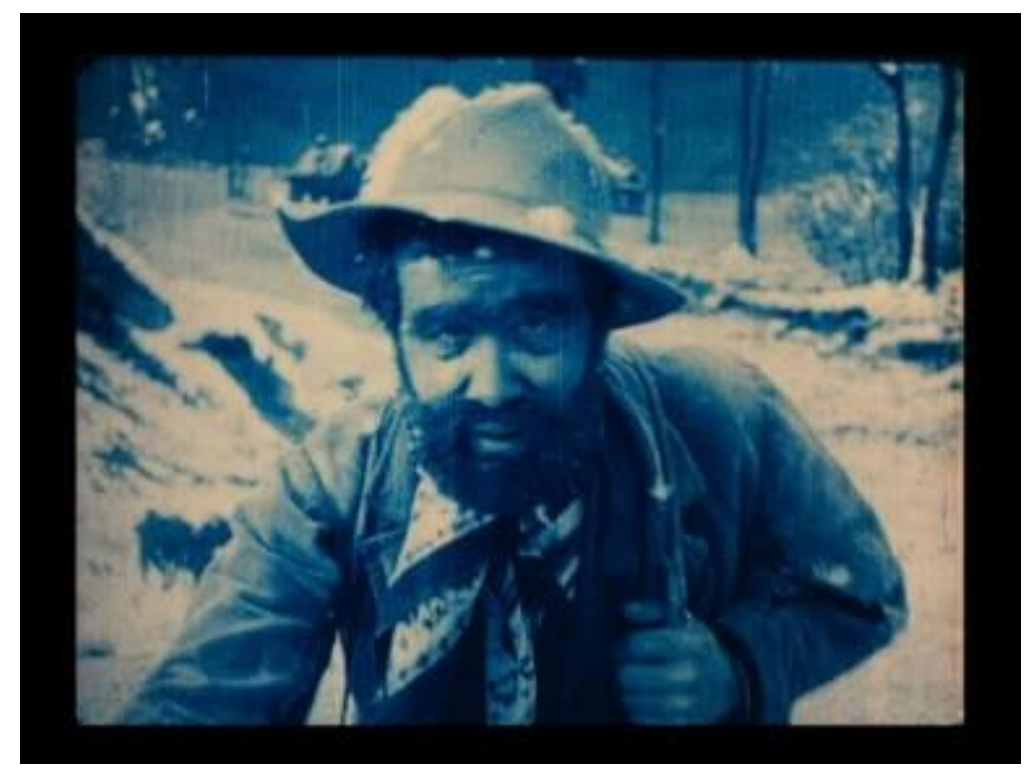

Fig. 1. Jean Valjean dans Le chemineau d'Albert Capellani, 1905.

Cette histoire de chandeliers et d'évêque aura un bel avenir cinématographique. C'est bien là le point de départ de la longue série d'adaptations américaines et françaises ( $\mathrm{y}$ compris la dernière en date de Tom Hopper). Peu d'adaptations des Misérables échappent à cette logique. ${ }^{4}$ Dans un matériau aussi dense que celui du roman, pourquoi ces scènes de la rencontre entre les deux hommes, le forçat et le clerc, forment-elle le noyau nucléaire des adaptations, le plus petit dénominateur commun entre toutes ? Sans doute, sont-elles essentielles à la construction du personnage de Valjean et à sa "rédemption ". Mais pourquoi s'arrêter en si bon chemin et ne pas utiliser la scène du vol du Petit Gervais, qui constitue dans le roman le moment de bascule dans la destinée du forçat : c'est ce crime commis contre un enfant qui réveille la conscience de Jean Valjean et le pousse à quitter ce bagne permanent qu'est son passeport jaune, mais c'est aussi ce crime qui fera de lui « [ce] relaps, un forçat en rupture de ban » que Javert ne cesse de poursuivre.

Mais revenons-en au court-métrage de Capellani dont on a dit qu'il constituait le premier maillon de cette longue chaîne de réceptions cinématographiques des Misérables. Avec cette première adaptation fragmentaire du roman, c'est la peur du vagabond, du marginal, de ces crimes commis au plus profond de la nuit qui vient hanter les salles obscures. Dans son recueil d'articles intitulé Les ombres de l'histoire, Crime et Châtiment au 19ème siècle, I'historienne Michelle Perrot revient sur la construction de cette figure du vagabond, dans les années 1890/1910: «Le vagabond ne peut vivre que de prélèvements sur la nature ou la propriété, de cueillette ou de vol. Il braconne, chaparde ; au besoin, il tue » (318) et elle cite le Docteur Pagnier, dans son étude au titre sans ambiguïté, Un déchet social: le vagabond (1910): "Le vagabondage mène au crime [...] les criminels ont une véritable propension à errer... Presque tous les voleurs de profession sont des nomades, ils n'ont de goût que pour la vie d'aventure, la vie irrégulière » (ibid). Le court-métrage de Capellani - ou du moins la version que nous connaissons aujourd'hui - se conclut par l'arrestation de Valjean. Le retournement final de l'épisode - Myriel innocente Valjean et transforme le vol des chandeliers en don - est curieusement absent. En l'état actuel des connaissances sur ce film, il nous est difficile de savoir si un fragment reste manquant ou si le cinéaste a délibérément placé le spectateur face à l'alternative suivante : le contentement de voir le vagabond puni par les gendarmes ou le désir du spectateur de se raconter la suite de l'histoire. Le film est suffisamment transparent pour qu'un public familier du destin de Jean Valjean puisse se 
« remémorer » la séquence absente de l'écran - mais pas des esprits. Cette hypothèse est séduisante, mais elle serait moins opérante sur un public plus distant de l'œuvre originale. Là où Capellani a peut-être choisi l'ellipse, les premiers producteurs américains d'adaptations des Misérables choisiront l'emphase.

\section{The Bishop's Candlesticks}

Contemporains de Capellani, ces premiers films n'auront d'ailleurs pas pour titre Les Misérables (et encore moins Le Chemineau). II nous faut rappeler que parmi les premières adaptations américaines de l'ère du cinéma muet des Misérables, deux sont intitulées The Bishop's Candlesticks ${ }^{5}$ (Les chandeliers de l'évêque), et qu'une autre a pour titre Le pardon du forçat. ${ }^{6}$ Près de quarante années plus tard, soulignons que Lewis Milestone fait lui-même référence à son adaptation des Misérables sous cette dénomination. Se remémorant les conditions de production de son adaptation de 1953 dans un livre d'entretiens, Milestone confiait qu'il avait cherché à s'écarter du fil narratif dit des «Chandeliers de l'évêque » et qu'il en avait débattu avec ses producteurs dont le fameux Daryl Zanuck:

I pleaded with them: « there are fifteen other stories much better than "The Bishop's Candlesticks". Why do it again? The book has marvelous things. Take for instance, little Gavroche during the Revolution - this kid's story has something $"$.

They wouldn't listen. So, having exhausted all your intellectual arguments, you go in saying: « Oh, for Christ sake, it's just a job - l'll do it and get it over with. " ${ }^{7}$ (Higham et Greenberg, 191)

L'intention des premiers producteurs hollywoodiens se manifeste dans le choix des titres des versions muettes dénomination qui perdure au moins jusqu'aux années cinquante comme on le voit avec Lewis Milestone. Cette dimension est sans doute un peu oubliée car d'une part ces premiers films sont probablement perdus à jamais et d'autre part nous avons une inclinaison naturelle à penser que les films adaptés des Misérables ont immanquablement pour titre... Les Misérables, ce qui est généralement vrai dans les versions parlantes mais qui l'est moins dans les versions muettes, comme nous l'avons vu. De même, le titre d'une même version peut changer selon l'époque et/ou le pays d'exploitation.

Le film de Lewis Milestone - dont il faut remarquer qu'il était une production de second rang ${ }^{8}$ et certainement pas une adaptation prestigieuse dotée d'un budget important - avait pour titre Les Misérables aux États-Unis mais fut diffusé en France sous le titre La vie de Jean Valjean, avant de retrouver son titre original pour son exploitation contemporaine en vidéo et DVD. Le choix du titre français avait sans doute pour qualité d'être honnête - le scénario se concentre sur le destin de Valjean et élimine à peu près tous les autres éléments de l'intrigue - à défaut de l'efficacité commerciale : le film connût une diffusion modeste et fut un échec commercial en France. II faut bien reconnaître que le film de Milestone est en effet assez médiocre. Lewis Milestone était un cinéaste tout à fait capable, son adaptation du roman d'Eric Maria Remarque A l'Ouest rien de nouveau (1930) est un film marquant, mais sa carrière avait été pour le moins entravée par les effets dévastateurs de la guerre froide dans le monde des studios hollywoodiens. En effet, Milestone eut la mauvaise idée de réaliser pendant la Seconde guerre mondiale deux films "pro-soviétiques » Our Russian Front (1942) et The Northern Star (1943) et d'avoir par ailleurs la réputation d'être pacifiste et antinazi. Milestone fut placé sur la liste dite des "Hollywood 19 » en 1949 , il put continuer à travailler, ${ }^{9}$ mais sa marge de manœuvre face aux producteurs s'en trouve considérablement diminuée et il en est malheureusement réduit à faire le tâcheron pour des productions sans grande ambition. ${ }^{10}$ Bien que ses qualités esthétiques soient faibles - la mise en scène est académique, M. Rennie est un des Valjean les moins convaincants vus à l'écran -, le film de Lewis Milestone est néanmoins un objet d'études assez intéressant car il propose une lecture originale du roman. Les films historiques ou les adaptations ont souvent la particularité de porter un discours sur la société contemporaine, notamment dans le cinéma hollywoodien. La distance entre la société des spectateurs et celle représentée à l'écran - que cette distance soit culturelle, historique, géographique ou combine ces données - permet d'adopter plus facilement un discours critique mais aussi de donner une permanence et une universalité à un discours idéologique dominant spécifique.

Dans le cas de l'adaptation de Milestone, c'est cette deuxième option qui a été choisie. Jean Valjean nous est présenté comme un martyr chrétien puis comme un « self-made man ». L'ascension sociale de Valjean donne corps à deux aspects centraux de l'idéologie conservatrice américaine du début de vingtième siècle. L'association entre transformation morale - via la spiritualité chrétienne - et ascension sociale est un des éléments de ce que l'on a appelé "l'évangile de la richesse ». Ce courant théologique, dominant aux États-Unis dans la première partie du vingtième siècle, est notamment représenté par W. Lawrence, évêque du Massachusetts. Celui-ci écrivait, en 1901 : « c'est à l'homme moral uniquement qu'échoit l'opulence [...] la sainteté a partie liée à la richesse » ${ }^{11}$ (258). Alors qu'aux États-Unis, des voix ${ }^{12}$ s'élèvent contre les excès du capitalisme, des réponses religieuses et laïques soutiennent que cette forme de société est bénéfique aussi bien économiquement et socialement que moralement. 
Lewis Milestone illustre ces théories et pratique ainsi une réorientation idéologique du roman. En effet, Valjean apparaît dans cette adaptation comme profondément inspiré par le sentiment religieux - symbolisé par son attachement aux chandeliers, qui apparaissent à chacune de ces décisions importantes et qui marquent chacune des victoires de son sens moral - ; il est aussi celui qui ne part de rien et dont la réussite économique est à l'image de ces personnages mythiques des théories conservatrices américaines : les self made men. Le don des chandeliers conjugue chez Milestone la fusion des dimensions chrétiennes et économiques. II est intéressant de comparer trois dialogues tirés de trois versions américaines des Misérables, celle de Boleslawski en 1933 et celle de Billie August en 1998 s'ajoutant à celle de Lewis Milestone. Les trois films ont en commun de faire de l'intervention de Myriel l'évènement qui permet de soustraire Valjean à une nouvelle condamnation et marque le début d'un nouveau chapitre de sa destinée: " take them [l'argenterie] freely. And use them wisely, so that you will never have to go to the galley $"{ }^{13}$ dit le Myriel de Milestone; " Don't forget your promise to be a new man, [...] you no longer will belong to evil. I've ransomed you from fear and hatred " ${ }^{14}$ déclare celui de Billie August; "let me give and in return, promise me you'll give also " ${ }^{15}$ sont les dernières paroles de l'évêque de la version de Boleslawski. La différence entre le discours du Myriel de Milestone et ceux d'August et Boleslawski : l'argenterie de l'évêque est l'objet d'un don/contredon chez ces deux derniers. Chez Myriel, les chandeliers sont essentiellement une valeur d'échange (au sens économique du terme). Avec ce capital, c'est la promesse de ne plus aller en prison qui se réalise. Néanmoins, si l'on se réfère à "l'Évangile de la richesse " que nous évoquions plus haut, il ne saurait y avoir de contradiction entre les deux termes : en devenant prospère Valjean deviendra un homme meilleur. En outre, le film de Milestone propose une modification radicale d'un des épisodes clés du roman. Le vol du Petit-Gervais, que nous avons évoqué plus haut, est la dernière manifestation de la brutalité du bagnard. Ce qui fait sortir Valjean de l'hébétude dans laquelle 20 ans de bagne l'ont plongé, ce n'est pas la bonté de Myriel, c'est le désespoir de l'enfant qu'il vole d'une pièce de 5 francs. Cet épisode est souvent éludé dans les adaptations. Milestone le transforme complètement. Après son passage chez Myriel, Valjean se rend dans une ville - nous apprendrons qu'il s'agit de Montreuil - et y sauve un enfant d'une mort probable provoquée par l'emballement des chevaux de son attelage. Au refus d'entendre la plainte de Petit-Gervais du Valjean de Victor Hugo se substitue un Valjean risquant sa vie pour les cris d'un enfant. Par suite de ce sauvetage, Valjean gagne une reconnaissance sociale symbolisée par une nouvelle désignation : il était « yellow passport convict », il devient « Sir ».

\section{Valjean : un self-made man ?}

La réussite économique de Valjean n'est pas, elle, une invention de Lewis Milestone. En effet, sous le nom de Monsieur Madeleine, Valjean devient dans le roman un industriel qui bâtit rapidement une fortune importante. À la différence du roman, le film de Milestone, cependant, omet de montrer la misère, qui est le sort de la très grande majorité des Misérables, et notamment de Fantine, ouvrière de Monsieur Madeleine, dont le destin tragique jette une ombre sur le paradis social du bienfaiteur de la petite ville de Montreuil. En occultant la représentation de cette misère, Milestone transforme l'exception Valjean en une possibilité Valjean ouverte à tous.

Mais le film de Milestone va plus loin dans l'illustration des théories économiques à la mode de son temps. Une scène se déroule dans la petite fabrique de vaisselle que Valjean visite par hasard avant de l'acheter. Certes, l'usine ne respire pas la prospérité mais cela est principalement dû à la mauvaise organisation de la production. Valjean décide de réorganiser celle-ci : alors qu'auparavant chaque ouvrier avait la maîtrise de toutes les étapes de la production de la vaisselle, Valjean décide de mettre en place le travail à la chaîne et la spécialisation des tâches. Cette réorganisation, fordiste avant l'heure, est évidemment anachronique. Mais elle conduit, dans le film de Milestone, à l'épanouissement économique ; elle apparaît ainsi comme une illustration de la supériorité de ce modèle économique, une justification d'un modèle que Sean Dennis Cashman décrit ainsi : " The Ford assembly line achieved enormous increase in productivity by transferring the decision as to the pace of work away from the workers, and into the hands of the manager who controlled the product line ${ }^{16}(221)$.

L'écart entre le film de Milestone et le livre est à souligner : pour Victor Hugo, l'intervention de Valjean permet aussi de sauver l'entreprise en difficulté toutefois elle ne concerne pas la façon de produire mais le choix des matières premières : "Cette industrie avait toujours végété, à cause de la cherté des matières premières. [...] un homme [...] avait eu l'idée de substituer, dans cette fabrication, la gomme-laque à la résine [...]. Ce tout petit changement en effet avait réduit le prix de la matière première » (Les Misérables I, V, $1: 183-184)$.

Ainsi, dans le film, l'histoire de Valjean devient celle d'une figure centrale du conservatisme : « au confluent de la morale et de l'évolutionnisme, le mythe du "self made man », fils et père ${ }^{17}$ de ses œuvres, légitime le succès de quelques-uns, en entretenant les espoirs de tous » (Martin, Royot et Bourget 260). Ce personnage du "self made man » est à la fois présent dans les travaux théoriques conservateurs pour qui « la société américaine prouve chaque jour la validité du « socio-darwinisme » en permettant l'ascension en haut de la pyramide sociale de ceux, qui 
nés à la base, en ont reçu les moyens de Dieu et de la nature » (ibid), mais aussi dans une littérature populaire édificatrice avec l'abondante production d'Horatio Alger (1832-1899), piètre pasteur puis auteur à succès, dont le Dick en haillons, le Tom en lambeaux, et toute la série "Chance et Courage " («Luck and Pluck » series) se vendent par millions d'exemplaires et popularisent encore le mythe de l'ascension, « des loques à l'opulence » ( from rags to riches $»)$.

Contrairement au roman, le Valjean de Milestone n'est pas un homme seul. Dans son adaptation, Robert, (un personnage absent du roman) contremaître de la fabrique achetée par Valjean, devient son associé. Cet apport vise deux objectifs, le premier étant de créer un double laïc au personnage de Myriel. Robert est un citoyen ordinaire et honnête : en acceptant celui qu'il devine être un ancien forçat, il devient le symbole du droit à une vie normale pour Valjean. Lui aussi est en quelque sorte un justicier, car comme Myriel, il s'oppose à l'injustice en aidant Valjean et en participant à son entreprise de "rachat " individuel - un des enjeux du film comme le souligne son carton d'introduction : "Forçat libéré, Jean Valjean veut se racheter ». II symbolise aussi l'insertion permanente de Valjean dans une communauté. En effet, contrairement au roman où Valjean cède son entreprise à ses ouvriers, Robert lui permet de garder le contrôle de ses affaires et finalement de continuer à être le bienfaiteur de Montreuil.

Arrêtons-nous un instant sur ce tour de passe-passe. Le lecteur du roman sait peu de choses de la postérité de l'usine de Madeleine, si ce n'est que l'entreprise périclite. Faut-il y voir la condamnation du projet utopique de Madeleine ? II faudrait évidemment plus que cet article pour réfléchir à cette question. Nous nous risquerons néanmoins à deux hypothèses. Cet échec pourrait être un commentaire des projets socialiste-utopiques, du type de ceux des phalanstères de Charles Fourier et Victor Considérant. Une autre hypothèse - non contradictoire avec la précédente - nous inviterait à une compréhension plus large du personnage de Jean Valjean. Ce dernier ne serait en effet ni un réformateur ni un révolutionnaire, il est hors de l'histoire. II crie "Je suis Jean Valjean », mais ce cri se perd et il disparaît comme a disparu son œuvre philanthropique. Condamné à l'anonymat, il ne laisse pas de traces, y compris jusqu'après sa mort : "Cette pierre [la pierre tombale de Valjean] est toute nue. [...] On n'y lit aucun nom. Seulement, voilà de cela bien des années déjà, une main y a écrit ces quatre vers qui sont devenus peu à peu illisibles sous la pluie et la poussière, et qui probablement sont aujourd'hui effacés " ${ }^{18}$ (Les Misérables V, IX, $6: 300$ ).

En tous les cas, Montreuil ne connaît plus la prospérité : « Lui tombé, chacun tira à soi ; l'esprit de lutte succéda à l'esprit d'organisation, la haine de l'un contre l'autre à la bienveillance du fondateur pour tous ; [...] le salaire baissa, les ateliers chômèrent, la faillite vint. Et puis plus rien pour les pauvres. Tout s'évanouit » (Ibid, II, II, 1 : 392).

À cette vision profondément pessimiste du roman s'oppose dans le film de Milestone une thématique typiquement

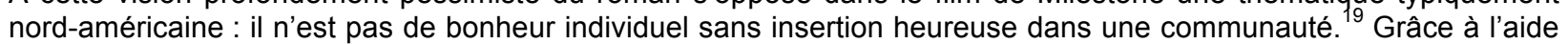
de son ami Robert, Valjean est un acteur déterminant de la prospérité de cette communauté.

De plus, ce personnage de Robert symbolise lui aussi l'ascension sociale : son statut d'ouvrier - bien qu'il faille noter qu'il appartient plutôt à une forme d'aristocratie ouvrière - se transforme en un statut de bourgeois négociant.

La requalification du destin de Valjean en tant que « success story » est un indice de l'utilisation du cinéma, cet art populaire par excellence, comme véhicule idéologique de formes du conservatisme nord-américain.

Pour le film de Milestone, l'occultation d'un pan de la dimension contestataire du roman - la volonté de redistribution et le refus de la misère - est le résultat de la situation économique florissante des États-Unis et le poids du Maccarthysme sur Hollywood. Milestone respecte la norme idéologique de son temps. Se faisant, il se positionne de façon fort différente de son prédécesseur Richard Boleslawski, dont l'adaptation aura elle pour originalité de se concentrer sur la critique des conditions de détentions des bagnards, revisitant la trame romanesque d'une précédente production; I Was A Prisonner From A Chain Gang. ${ }^{20}$ Mais le contexte de production des deux films s'est profondément modifié. Le film de Milestone est diffusé dans une période historique bien différente de celle de l'adaptation de Boleslawski : la grande dépression des années trente est oubliée, l'après-guerre est une période de «boom» économique pour les États-Unis. Les attentes des classes moyennes et populaires en sont considérablement modifiées, comme le souligne Norbert S. McElvaine : « During times of relative prosperity, many in the middle class have tried to emulate them above on the social scale and so adopted their values: [...] the acquisitive ethic has dominated. The twenties, the fifties and the seventies are leading examples of such periods » ${ }^{21}(203)$.

Les adaptateurs américains des Misérables ont bien compris que le roman de Victor Hugo ne se réduisait pas à une unique trame narrative. Comme le remarquait Lewis Milestone, il y a 15 histoires dans le roman. Cette approche 
Szabo, Fabrice. " "The Bishop's Candlesticks" : une certaine tradition de la réception hollywoodienne des Misérables. » Nouvelle Revue Synergies Canada, №13 (2020)

rompt avec le dyptique fidélité/infidélité qui prévaut le plus souvent dans la critique et chez les adaptateurs français, surtout lorsqu'il s'agit d'œuvres «patrimoniales » comme le roman hugolien. Ces préoccupations étaient peu présentes dans l'esprit des adaptateurs américains qui s'intéressent au film à faire en tenant compte des contraintes internes du système de production - l'esthétique propre à chaque studio, le système industriel hollywoodien qui implique de s'appuyer sur telle équipe de techniciens, tels comédiens, tel scénariste ou encore le Code de Production qui pose les limites et les interdits à respecter à l'écran ${ }^{22}$ - et de contraintes externes que sont les attentes et les compétences du public ou encore le contexte socio-politique. Tout ceci converge à faire des films hollywoodiens des œuvres collectives dans lesquelles le concept de « film d'auteur » est essentiellement inopérant. C'est par une sorte de facilité de langage que nous avons souvent mentionné " le film de Lewis Milestone », il aurait sans doute été plus juste de parler du « film réalisé par Lewis Milestone dans le respect de la commande de son producteur Daryl Zanuck et à l'ombre des investigations du HUAC ». ${ }^{23}$

En effet, Milestone aurait aimé que son film s'appuie sur une autre histoire que celle des « Bishop's Candlesticks ». Et pourtant, c'est bien là le film qu'il va réaliser tout en y ajoutant une dimension économique en appuyant le portrait d'un Jean Valjean, self-made man. Ainsi, il contribue malgré lui - mais de manière originale - à la tradition hollywoodienne de réception du roman dans laquelle les chandeliers occupent une double-fonction. D'une part, elles supplantent Jean Valjean dans l'ordre symbolique de la narration: Les Misérables, ce n'est pas la vie de Jean Valjean mais le destin d'une paire de chandeliers. L'objet prend le pas sur le(s) personnage(s). Cet enrichissement est particulièrement intéressant et vient compléter la réflexion que nous offrait Michel Serceau autour du « personnage-signe » dans certaines adaptations, dont celles des romans de Victor Hugo :

Les adaptations les plus factuelles, celles des mélodrames, des romans d'Alexandre Dumas, de Victor Hugo... accentuent le système actantiel et surtout, au sein de ce système, la fonction d'un personnage central. A tel point qu'il prend une dimension quasiment mythique [...] Le personnage-signe peut donc acquérir, paradoxalement, non pas une dimension subjective ni à proprement parler un poids de conscience, mais le pouvoir de cristalliser un état de conscience. (134)

Avec «les Chandeliers de l'évêque » nous pouvons envisager que le concept de "personnage-signe » soit compléter par celui d' " objet-signe ". Ils sont dès lors plus que des "objets ", ils deviennent l'essence même de la narration. Imaginons un instant que l'on demande à un réalisateur de raconter "l'histoire de la barricade » ou bien « L'histoire de la poupée de Cosette » : ce serait sans doute là une autre façon de nous raconter le roman.

\section{Notes}

${ }^{1}$ Les Misérables, un film réalisé par Tom Hooper en 2012.

${ }^{2}$ Un drame en 5 actes présenté à la scène en 1897.

${ }^{3}$ Les Misérables, un film réalisé par Albert Capellani, avec Henry Krauss dans le rôle de Jean Valjean, produit par la S.C.A.G.L., distribué par Pathé (2h43 minutes).

${ }^{4}$ Signalons cependant deux films qui y dérogent: d'une part, l'adaptation soviétique de 1937 qui se concentre essentiellement sur le personnage de Gavroche et dont il aurait été très surprenant qu'un évêque fut le héros et d'autre part, l'adaptation télévisuelle de Marcel Bluwal qui elle se singularise par sa très longue ouverture consacrée à la représentation du Paris social et politique de 1832, Jean Valjean n'apparaissant à l'écran que plus de 40 minutes après le début du premier épisode ; un choix qui a dû déconcerter de nombreux téléspectateurs.

${ }^{5}$ The Bishop's Candlesticks de Herbert Breton (1913) et de Norman McKinnel (1929).

${ }^{6}$ L'auteur de ce film est Franck Lloyd (1918).

7 Je traduis. " Je les [les producteurs] ai supplié : « il y a quinze autres histoires bien meilleures que celle des Chandeliers de l'Évêque. Pourquoi en faire une autre version? Le livre a des choses incroyables. Prenez par exemple, le petit Gavroche pendant la Révolution. L'histoire de ce gamin vaut le coup. ". Ils n'ont pas voulu m'écouter, Donc, ayant épuisé tous vos arguments intellectuels, vous vous dîtes finalement: "Bon, après tout, c'est juste un boulot - je vais le faire à leur idée ».

${ }^{8}$ Le film est en noir et blanc, d'une durée de 100 minutes et Michael Rennie - habituellement acteur de second rôle dans les grandes productions - tient le rôle de Jean Valjean. Le casting de la version précédente de Richard Boleslawski (1933) reposait sur Frederic March qui était lui une star de premier plan puisqu'il était auréolé de l'Oscar du Meilleur Acteur pour l'année 1931. 
Szabo, Fabrice. " "The Bishop's Candlesticks" : une certaine tradition de la réception hollywoodienne des Misérables. » Nouvelle Revue Synergies Canada, №13 (2020)

\footnotetext{
${ }^{9}$ Il connut néanmoins un exil temporaire et réalisa quelques films en Europe au milieu des années 50.

${ }^{10}$ Heureusement pour lui la fin de sa carrière connut un certain regain créatif puisqu'il la conclut par trois films dignes d'intérêt : Pork Chop Hill - un film sur la guerre du Corée où il souligne le pessimisme des combattants retrouvant la veine d'A l'Ouest Rien de Nouveau -, la version originale d'Ocean Eleven avec Frank Sinatra et enfin il fut enfin crédité pour le remake de Mutiny on the Bounty avec Marlon Brando. Le réalisateur Carol Reed a participé aux prises de vues (non-crédité) et la légende veut que Marlon Brando ait largement imposé ses choix de mise en scène.

11 Je souligne.

${ }^{12}$ Notamment du côté de certains théologues minoritaires comme Pierce qui raille les théories de Lawrence en les désignant comme « gospel of greed » (évangile de la cupidité).

13 Je traduis et souligne. «Prenez cela librement. Et utilisez-le avec sagesse, pour que jamais vous ne retourniez au bagne ».

14 Je traduis et souligne. « N'oubliez pas votre promesse d'être un autre homme [...] vous n'appartenez plus au mal. Je vous ai racheté à la peur et à la haine ».

15 Je traduis et souligne. "Laissez-moi vous donner, et promettez-moi que vous donnerez en retour ».

${ }^{16}$ Je traduis. « La méthode fordiste de travail à la chaîne réussit d'énormes gains de productivité en transférant la maîtrise du processus de production des employés vers les mains des dirigeants qui contrôlaient la chaîne ».

${ }^{17}$ L'association « fils et père » est par ailleurs un aspect central du Christ dans la théologie chrétienne.

18 Je souligne.

${ }^{19}$ L'écrivain américain Norman Mailler remarque à ce sujet que « les américains fonctionnent mieux en équipe. Peutêtre grâce à la conquête de l'ouest, parce qu'ils ont dû former des communautés » (Norman Mailler: une voix d'Amérique).

${ }^{20}$ I Was a Prisonner From a Chain Gang, réalisé par Mervyn LeRoy, Warner Bros, 1932, 93 minutes.

21 Je traduis. "Au cours des périodes de relative prospérité, nombreux parmi la classe moyenne essayèrent de s'élever sur l'échelle sociale et donc adoptèrent leurs valeurs [celles des classes supérieures]: le souci d'enrichissement dominait. Les années vingt, cinquante et soixante-dix sont des exemples particulièrement significatifs de telles périodes, au cours du vingtième siècle $»$.

${ }^{22}$ Ce code - plus familièrement connu sous le nom de Code Hays - prescrit de façon précise la conduite à tenir face à certains " thèmes » : le sexe, les rapports sociaux et la violence. En ce qui concerne les adaptations des Misérables, l'interdiction de représenter la prostitution pose évidemment problème dans le cas de Fantine.

${ }^{23}$ L'acronyme qui désigne la House Commitee on Un-American Activities.
}

\section{Bibliographie}

Cashman, Sean Dennis. America in the Twenties and Thirties: the Olympian Age of Franck Delano Roosevelt. New York University Press, 1989.

Clerc, Jeanne-Marie et Monique Carcaud-Macaire. L'adaptation cinématographique et littéraire. Klincksieck, 2004.

Higham, Charles et Joel Greenberg. The Celluloid Muse: Hollywood Directors Speak. New American Library, 1972.

Hugo, Victor. Les Misérables. Presses Pocket, coll. « Classiques », présentée et commentée par Arnaud Laster, 1998 , t. I : 615 p., t. II : 620 p., t. III : 451.

Hutcheon, Linda. A Theory of Adaptation. Routledge, 2006.

I Was a Prisonner From a Chain Gang. Réalisé par Mervyn LeRoy, Warner Bros, 1932, 93 minutes. 
Szabo, Fabrice. " "The Bishop's Candlesticks" : une certaine tradition de la réception hollywoodienne des Misérables. " Nouvelle Revue Synergies Canada, N¹3 (2020)

Lawrence, W. « Les rapports de la richesse à la morale », Histoire de la Culture américaine. P.U.F., coll. « Premier Cycle », 1993.

Le Chemineau. Court-métrage réalisé par Albert Capellani, Pathé Frères, 1905, 5 minutes.

Les Misérables. Réalisé par Albert Capellani, avec Henry Krauss dans le rôle de Jean Valjean, S.C.A.G.L., distribution Pathé, 1913, 163 minutes.

Les Misérables. Réalisé par Richard Boleslawski, avec Fredric March dans le rôle de Jean Valjean, 20th Century Pictures, 1935, 108 minutes.

Les Misérables. Réalisé par Lewis Milestone, avec Michael Rennie dans le rôle de Jean Valjean, 20th Century Fox, 1952, 101 minutes.

Les Misérables. Réalisé par Billie August, avec Liam Neeson, Mandalay Entertainment, 1998, 134 minutes.

Martin, J.P, D. Royot, et J-L. Bourget. Histoire de la Culture américaine. P.U.F., coll. « Premier Cycle », 1993.

McElvaine, Robert S. The Great Depression. Times Books, 1984.

Norman Mailler : une voix d'Amérique. Réalisé par Richard Copans et Stan Newmann, 1999.

Perrot, Michèle. Les ombres de l'histoire, Crime et Châtiment au 19ème siècle. Flammarion, 2001.

Rosa, Guy. "Histoire sociale et roman de la misère ", communication disponible sur le site du Groupe Hugo de I'Université Paris-VII : http://groupugo.div.jussieu.fr/Default_Etudes.htm, 1995.

Serceau, Michel. L'adaptation cinématographique des textes littéraires : Théorie et lecture. Édition du CEFAL, 1999. 